\title{
Biosensing by Learning: Cancer Detection as Iterative Optimization
}

\author{
Y. Chen*, N. Sharifi, G. Holmes \\ Faculty of Computing and Mathematical \\ Sciences \\ University of Waikato \\ Hamilton, New Zealand \\ *yifan.chen@waikato.ac.nz
}

\author{
U K. Cheang \\ Department of Mechanical and Energy \\ Engineering \\ Southern University of Science and Technology
Shenzhen, China \\ Southern University of Science and Technology
Shenzhen, China
}

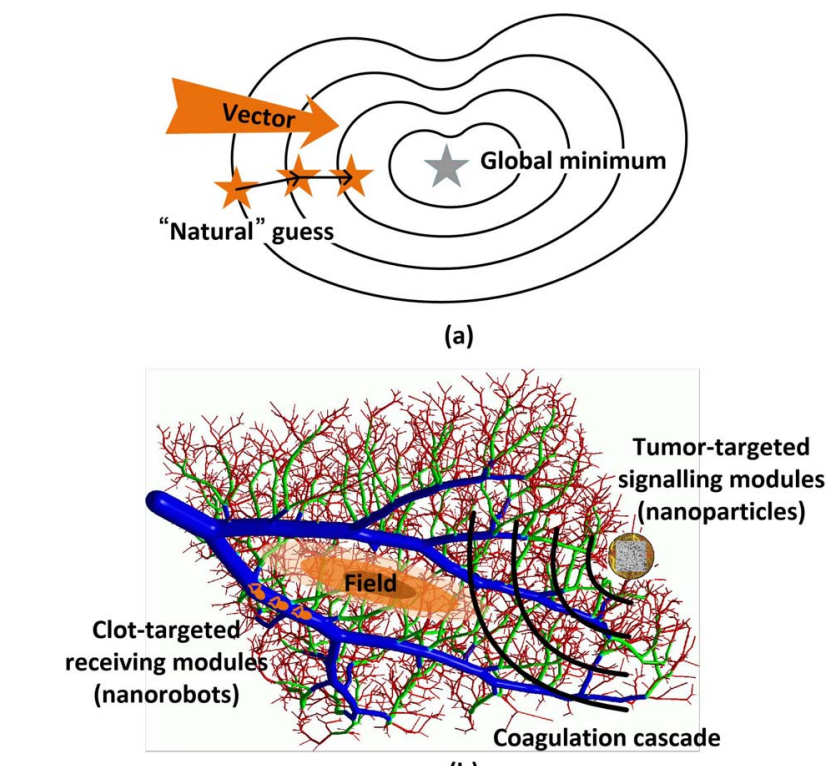

(a)

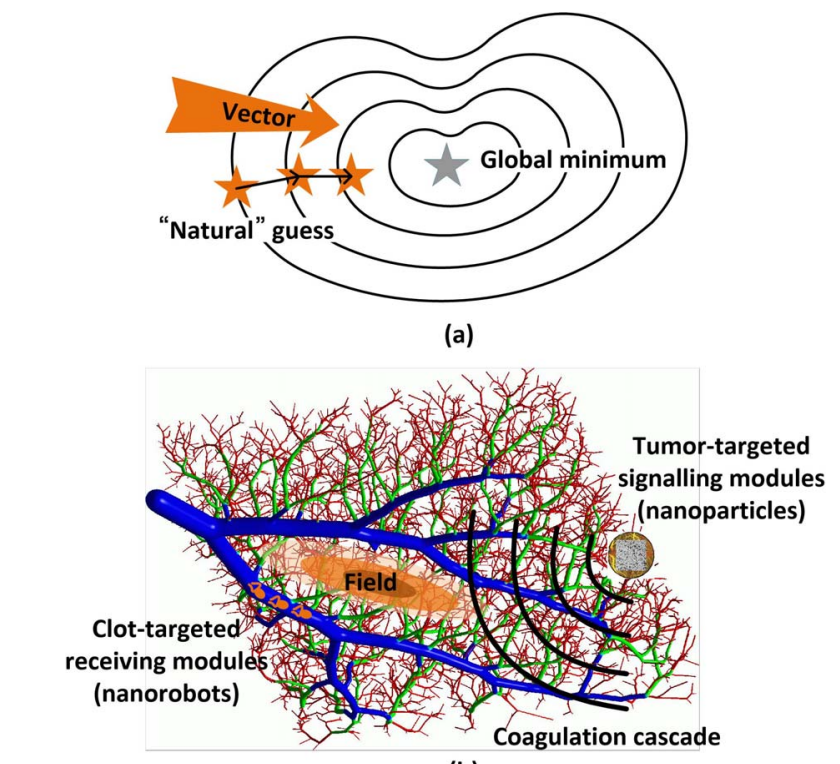

(b)

Fig. 1. Analogy between (a) iterative optimization and (b) externally controllable cancer detection.

Nature's blueprints have inspired exciting new fields of science such as bio-inspired computing that creates problemsolving techniques using insights from natural systems. For example, the process of natural selection inspired the development of the classical genetic algorithm to solve complex optimization and search problems. It is also stimulating to "look the other way" by exploiting computing strategies for biomedical applications [1], [2]. There is an intriguing analogy between iterative optimization and externally controllable cancer detection as depicted in Fig. 1. The global minimum of a smooth, unimodal biological cost function associated with a tumor-induced in vivo phenomenon with tumor being the epicenter is the cancer to be detected; the domain of the cost function is the tissue region at high risk of malignancy; the guess solution is a swarm of externally manoeuvrable nanorobots such as magnetotactic bacteria [3] or magnetofluorescent iron oxide nanoworms [4]. The guess input (i.e., nanorobots) locates the optimal solution (i.e., cancer) by moving through the domain (i.e., high-risk tissue) under the guidance of a specified vector (i.e., steering field) as shown in Fig. 1. The cost function may be altered by the guess made of natural materials because the guess input interacts with the domain (i.e., nanorobots undergo

\begin{abstract}
We propose a novel cancer detection procedure (CDP) based on an iterative optimization method. The global
minimum of a tumor-induced biological cost function indicates the tumor location, the domain of the cost function is the guess input is a swarm of externally controllable and trackable nanorobots for tumor sensing. We consider the spatial distrib-
ution of fibrin as the cost function; the fibrin is formed during the coagulation cascade activated by tumor-targeted signalling modules (nanorobots) towards the site of disease. Subsequently, the CDP can be interpreted from the iterative optimization continuously updated according to the gradient of the cost function in order to find the optimum (i.e., cancer) by moving
through the domain (i.e., tissue under screening). Along this line of thought, we consider the gradient descent (GD) iterative
method, and propose the GD-inspired CDP, which takes into account the realistic in vivo propagation scenario of nanorobots.
\end{abstract}

\section{INTRODUCTION} cont

physical, chemical, and biological reactions in the in vivo environment). This is in contrast to a traditional iterative method using a non-interacting approximate solution. An external observer can then infer the domain by monitoring the movement of the guess ("seeing-is-sensing" [1]), where the $(n+1)$ th approximation is derived from the $n$th one. This strategy is within the general framework of computinginspired bio-detection proposed in our previous work [2].

Provided with the analogy, a wide variety of iterative methods can thus be applied to the design of optimal cancer detection procedures (CDPs). It has been shown in [4] that nano-systems can communicate to amplify disease targeting in vivo. These systems are composed of signalling modules that target tumors and then locally activate the coagulation cascade to broadcast tumor location to clot-targeted receiving modules in circulation that carry a diagnostic or therapeutic cargo, thereby amplifying their delivery. Hence, it is plausible to use the distribution of fibrin formed through the cascade reaction as the cost function. To elaborate on 
the proposed technique, the classical gradient descent (GD) method is used to inspire the CDP, where the guess input takes steps based on the gradient of the cost function at the current point. Furthermore, the derivative of the function needs to be approximated in real-time and the movement of the guess is constrained by the physical conditions of human microvasculature.

The paper is organized as follows. In Section II, we introduce the general iterative bio-detection framework. In Section III, we propose the GD-inspired CDP. In Section IV, we provide numerical examples to demonstrate the effectiveness of the proposed framework. Finally, some concluding remarks are drawn in Section V.

\section{ITERATIVE BIO-DETECTION}

To elaborate on the proposed biosensing-by-learning framework, we consider the nano-communication system proposed in [4], wherein signalling modules would first target tumors and then broadcast the tumor's location to receiving modules in circulation as shown in Fig. 1(b). The coagulation cascade was selected due to its powerful signal amplification, positive feedback, ubiquitous presence in plasma, and potential to operate across multiple tumor types. We focus on the following scheme. The signalling modules are nanoparticles (gold nanorods) that target tumors and convert external electromagnetic energy into heat in order to locally disrupt tumor vessels and initiate extravascular coagulation. This also results in a temperature gradient from the tissue malignancy to its peripheral region. Consequently, the fibrin forms the "coagulation" gradient centered at the tumor caused by temperature increase. The receiving modules are magnetofluorescent iron oxide nanoworms, which employ the peptide coatings that recognize fibrin directly for clot targeting and can be controlled and tracked externally.

Consider a cost function $f$ representing the spatial distribution of fibrin after activation of the extravascular coagulation pathway upon binding of signalling modules to tumor receptors. The cost function is defined on the domain $\mathbb{D}$ representing the high-risk tissue region under surveillance. The landscape of $f$ is distorted by the "natural" guess $G$ representing a swarm of receiving modules as follows

$$
\begin{aligned}
f(\vec{x} ; G) & =f_{\mathrm{M}}(\vec{x} ; G)+f_{\mathrm{C}}(\vec{x} ; G) \\
& =f_{\mathrm{I}}(\vec{x})+f_{\mathrm{E}}(\vec{x} ; G)+f_{\mathrm{C}}(\vec{x} ; G), \quad \vec{x} \in \mathbb{D},
\end{aligned}
$$

where $f_{\mathrm{M}}(\vec{x} ; G)$ is the externally measurable function at location $\vec{x}$ through guess $G, f_{\mathrm{C}}(\vec{x} ; G)$ is the correction factor accounting for the fitness of $G$, denoted by $\mathcal{F}_{G}$, due to its degradation in $\mathbb{D}, f_{\mathrm{I}}(\vec{x})$ is the intrinsic cost function at $\vec{x}$ independent of the presence or absence of $G, f_{\mathrm{E}}(\vec{x} ; G)$ is the extrinsic disturbance caused to the function due to the interaction between $G$ and the domain $\mathbb{D}$. For a meaningful optimization process, it is assumed that regardless of any variation caused by the guess to the cost function, the location of the global maximum denoting the tumor, $\vec{x}^{\star}$, remains unchanged.

The intrinsic function $f_{\mathrm{I}}(\vec{x})$ should reflect the concentration of fibrin in circulation. As each swarm of receiving modules would diffuse during propagation and clot targeting assists in local aggregation, it is assumed that $f_{\mathrm{I}}(\vec{x})$ is proportional to the difference between the expected reduction in the fluorescent quantification of receiving modules due to diffusion alone [7] and the actual fluorescence reduction due to the combined effect of diffusion and coagulation targeting at $\vec{x}$. The extrinsic disturbance $f_{\mathrm{E}}(\vec{x})$ is the fluorescence reduction resulting from the interaction between receiving modules and human vasculature at $\vec{x}$ (i.e., degradation of nanorobots). The correction factor $f_{\mathrm{C}}(\vec{x} ; G)$ compensates for the degradation. Finally, typical skeletonized images of vascular networks demonstrate that normal capillaries produce essentially two-dimensional patterns such that they are almost uniformly distributed to ensure adequate oxygen transportation throughout the tissue [5]. On the other hand, the microvascular density in the peritumoral region increases due to the supply of growth factors from the tumor and reduces in the tumor center due to a combination of severely reduced blood flow and solid stress exerted by the tumor [6]. Hence, we assume that the normal tissue is regularly vascularized with a homogeneous capillary network of given microvascular density that is fixed by intercapillary distance. The density increases at the tumor periphery and reduces inside the tumor. The overall cost function $f(\vec{x} ; G)$ is thus discontinuous over $\vec{x}$ caused by the grid structure of vascular networks.

Subsequently, a guess input $G$ is randomly distributed in a pre-specified deployment region $\mathbb{R} \subseteq \mathbb{D}$. Depending on the availability of prior knowledge about the cost function, the guess begins searching for a solution following some iterative algorithm. The sequence of the optimization process is as follows.

1) Initialization. The guess input is deployed in $\mathbb{R}$ at the starting time $t_{0}$ with initial locations $\vec{x}\left(t_{0}\right)$. We define the fitness of $G$ to be the duration when $G$ has been interacting with $\mathbb{D}$, as the deterioration level of the guess increases with the time of operation. The initial fitness $\mathcal{F}_{G}\left(t_{0}\right)=0$.

2) Updating. The trajectory of the guess is determined by the angle deviation relative to a principal axis denoting the external vector upon $G$ at $\vec{x}\left(t_{0}\right), \phi\left(\vec{x}\left(t_{0}\right)\right)$, and the displacement length, $d\left(t_{0}\right)$, which depend on the iterative method described in Section III. The next location of the guess at time instant $t_{1}$ is then updated according to:

$$
\vec{x}\left(t_{1}\right)=\vec{x}\left(t_{0}\right)+d\left(t_{0}\right) \vec{u}_{\angle \phi\left(\vec{x}\left(t_{0}\right)\right)}+\vec{\delta}\left(t_{0}\right)
$$

where $\vec{u}_{\angle \phi}$ is a unit vector with angle $\phi$ and $\vec{\delta}\left(t_{0}\right)$ is the position updating error due to the physical constraint of the microvasculature.

3) Cost Function Evaluating. In contrast to a traditional iterative method where an exact knowledge of the updated guess input is available, the new position of the guess needs to be estimated for bio-detection due to positioning inaccuracy. The approximated new location of the guess is given by $\vec{x}\left(t_{1}\right)+\vec{\zeta}\left(t_{1}\right)$ with $\vec{\zeta}\left(t_{1}\right)$ being 

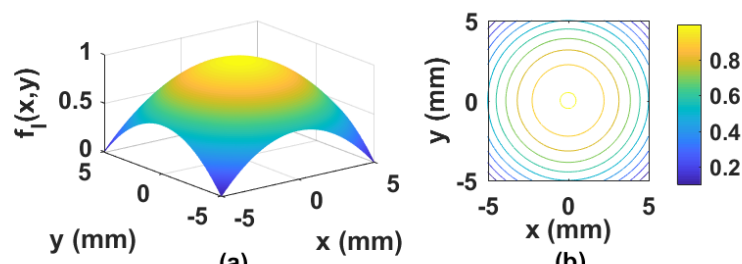

(b)
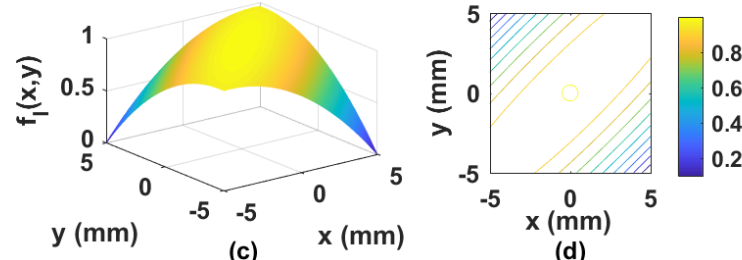

(d)

Fig. 2. Illustration of $f_{\mathrm{I}}(\vec{x})$, which corresponds to the difference between the expected reduction in the fluorescent quantification of receiving modules due to diffusion alone and the actual fluorescence reduction due to the combined effect of diffusion and coagulation targeting at $\vec{x}$. The maximum is normalized to 1 and the minimum value is 0 indicating no clot-targetinginduced aggregation of nanorobots: (a) Sphere function and (b) its contour plot; (c) Matyas function and (d) its contour plot.

the localization error. The cost function is then evaluated by monitoring the state of $G$ from $t_{0}$ to $t_{1}$. The updated fitness of $G$ is $\mathcal{F}_{G}\left(t_{1}\right)=t_{1}-t_{0}$.

4) Decision-making and Iterating. Following from (1), the value of the cost function obtained by the guess is computed. If certain stopping criteria are met, the searching is terminated. Otherwise, a new vector for the guess is generated by following some iterative algorithm. The displacement vector $d\left(t_{1}\right) \vec{u}_{\angle \phi\left(\vec{x}\left(t_{1}\right)\right)}$ determines the subsequent location of $G$ as described in (2). In the case that a specific guess fully degenerates in $\mathbb{D}$, a new guess input is deployed at $\mathbb{R}$. Subsequently, the iteration continues.

As an initial investigation, we assume that the effect of degradation has been exactly counterweighted, i.e., $f_{\mathrm{E}}(\vec{x} ; G)=-f_{\mathrm{C}}(\vec{x} ; G)$ and $f(\vec{x} ; G)=f_{\mathrm{I}}(\vec{x})$. Two representative cost functions are considered to evaluate the cancer detection performance as shown in Fig. 2, where the maximum value is normalized to 1 and the minimum value is 0 denoting the situation that there is no aggregation of nanorobots due to clot targeting. The search domain is $-5 \mathrm{~mm} \leq x, y \leq 5 \mathrm{~mm}$.

1) Sphere Function (Bowl-shaped):

$$
f_{\mathrm{I}}(\vec{x})=\left\{\begin{array}{lr}
1, & \sqrt{x^{2}+y^{2}} \leq 0.5 \\
1-0.02\left(x^{2}+2 y^{2}\right), & \text { Otherwise. }
\end{array}\right.
$$

2) Matyas Function (Plate-shaped):

$$
\begin{aligned}
& f_{\mathrm{I}}(\vec{x})= \\
& \left\{\begin{array}{lr}
1, & \sqrt{x^{2}+y^{2}} \leq 0.5 \\
1-0.0104\left(x^{2}+y^{2}\right)+0.0192 x y, \text { Otherwise. }
\end{array}\right.
\end{aligned}
$$

As shown in Fig. 2, both the Sphere and Matyas functions represent the situation that the core of a cancer, denoted by a circle of radius $0.5 \mathrm{~mm}$ centered at the origin, exhibits fibrin tropism and pronounced homing of nanorobots. The

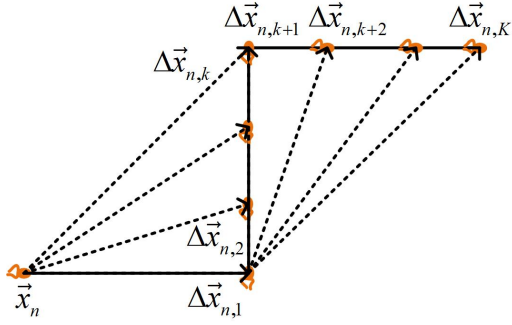

Fig. 3. Pictorial illustration of the Gradient Estimation process: the initial location of the $n$th cycle is $\vec{x}_{n}$; the guess moves towards various locations along a pre-specified zigzag pathway, $\vec{x}_{n}+\Delta \vec{x}_{n, 1}, \cdots, \vec{x}_{n}+$ $\Delta \vec{x}_{n, k}, \vec{x}_{n}+\Delta \vec{x}_{n, 1}+\Delta \vec{x}_{n, k+1}, \cdots, \vec{x}_{n}+\Delta \vec{x}_{n, 1}+\Delta \vec{x}_{n, K}$; the gradient is approximated as $\nabla f_{\mathrm{I}}\left(\vec{x}_{n}\right) \approx \max \left\{\left[f_{\mathrm{I}}\left(\vec{x}_{n}+\Delta \vec{x}_{n, 1}\right)-\right.\right.$ $\left.f_{\mathrm{I}}\left(\vec{x}_{n}\right)\right] /\left\|\Delta \vec{x}_{1}\right\|, \cdots,\left[f_{\mathrm{I}}\left(\vec{x}_{n}+\Delta \vec{x}_{n, k}\right)-f_{\mathrm{I}}\left(\vec{x}_{n}\right)\right] /\left\|\Delta \vec{x}_{k}\right\|,\left[f_{\mathrm{I}}\left(\vec{x}_{n}+\right.\right.$ $\left.\left.\Delta \vec{x}_{n, 1}+\Delta \vec{x}_{n, k+1}\right)-f_{\mathrm{I}}\left(\vec{x}_{n}+\Delta \vec{x}_{n, 1}\right)\right] /\left\|\Delta \vec{x}_{k+1}\right\|, \cdots\left[f_{\mathrm{I}}\left(\vec{x}_{n}+\Delta \vec{x}_{n, 1}+\right.\right.$ $\left.\left.\left.\Delta \vec{x}_{n, K}\right)-f_{\mathrm{I}}\left(\vec{x}_{n}+\Delta \vec{x}_{n, 1}\right)\right] /\left\|\Delta \vec{x}_{K}\right\|\right\}$ with equal and sufficiently small $\left\|\Delta \vec{x}_{n, 1}\right\|,\left\|\Delta \vec{x}_{n, k+1}\right\|$, and $\left\|\Delta \vec{x}_{n, K}-\Delta \vec{x}_{n, k+1}\right\|$.

aggregation of clot-targeted nanorobots decreases smoothly as the vessel becomes farther away from the core. Both the functions are convex and quadratic. They have no local minimum except the global one. For simplicity, we imprint the cost functions in (3) and (4) on the vascular network where the intercapillary distances are $100 \mu \mathrm{m}, 50 \mu \mathrm{m}$, and $200 \mu \mathrm{m}$ for normal $(1 \mathrm{~mm} \leq|x|,|y| \leq 5 \mathrm{~mm})$, peritumoral $(0.8 \mathrm{~mm} \leq|x|,|y| \leq 1 \mathrm{~mm})$, and tumoral $(|x|,|y| \leq 0.8 \mathrm{~mm})$ tissues, respectively [5]. The blood inflow and outflow are assumed to be in the bottom left and top right, respectively, where prescribed pressures are set.

\section{GD-INSPIRED CDP}

GD-inspired CDP starts with a guess $\vec{x}_{0}$ for a local maximum of $f_{\mathrm{I}}(\vec{x})$, and considers the sequence $\vec{x}_{0}, \vec{x}_{1}, \vec{x}_{2}, \cdots$ such that $\vec{x}_{n+1}=\vec{x}_{n}+\gamma_{n} \nabla f_{\mathrm{I}}\left(\vec{x}_{n}\right), n \geq 0$. We have $f_{\mathrm{I}}\left(\vec{x}_{0}\right) \leq f_{\mathrm{I}}\left(\vec{x}_{1}\right) \leq f_{\mathrm{I}}\left(\vec{x}_{2}\right) \leq \cdots$, so hopefully the sequence $\left(\vec{x}_{n}\right)$ converges to the desired local maximum. Suppose that $f_{\mathrm{I}}(\vec{x})$ is convex and $\nabla f_{\mathrm{I}}(\vec{x})$ is Lipschitz, the step size $\gamma_{n}$ can be chosen to guarantee convergence to a global optimum by using the Barzilai-Borwein method [8]. Note that the vessel network used in the simulation procedure is a discontinuous two-dimensional grid; therefore all the position vectors are mapped to the vectors associated with the nearest blood vessel. As the vessels run only parallel to the two coordinate axes, at each junction the guess input can move in two possible directions, up and right, as the flow is from bottom left to top right.

The standard GD algorithm is modified here to suit the CDP. Each cycle consists of multiple iterations discussed in Section II and can be divided into two processes: Gradient Estimation and Guess Update. In the former process, the guess moves towards various locations along a pre-specified zigzag pathway as depicted in Fig. 3. The gradient is then approximated to be the largest slope as described in the figure caption. In the latter process, the guess evolves according to the following equation:

$$
\vec{x}_{n+1}=\vec{x}_{n}+\gamma_{n} \cos \phi_{n} \vec{u}_{\mathrm{h}}+\gamma_{n} \sin \phi_{n} \vec{u}_{\mathrm{v}} .
$$


Eq. (5) describes the process that the guess is maneuvered towards the next location by first moving horizontally (i.e., unit vector $\vec{u}_{\mathrm{h}}$ ) and then vertically (i.e., unit vector $\vec{u}_{\mathrm{v}}$ ); $\phi_{n}$ is the angle of the gradient estimated at the $n$th cycle. Finally, due to the practical constraint of CDP, the initial deployment region of the guess input is confined within a small area, which is the injection site of nanorobots, instead of the entire solution space. To further ensure that the guess input is confined within the tissue region under screening, the replacement strategy is implemented: a guess that travels outside the allowed searching region is abandoned, which will degrade in the human body without further maneuvering and tracking. A new guess is then generated in the deployment area by injecting an aggregate of nanorobots.

\section{Performance Analysis}

We use several numerical examples to evaluate the performance of the GD-inspired CDP, which is compared to random search (i.e., the guess follows a random walk without learning from the environment). The two cost functions presented in Section II are applied to synthesize fibrin distributions giving rise to different levels of cancer detection difficulty. The initial deployment region is $-5 \mathrm{~mm} \leq x, y \leq$ $-4 \mathrm{~mm}$. The speed of nanorobots is $100 \mu \mathrm{m} / \mathrm{s}$. The maximum detection time allowed is $200 \mathrm{~s}$ and the total number of simulation runs is 1000 .

Fig. 4 shows the typical trajectories of guess inputs for different scenarios. For both the Sphere and Matyas functions, the GD-inspired CDP successfully detects the core of the cancer by using only one guess input (i.e., a single swarm of nanorobots). On the other hand, the random search technique fails to find the center of tissue malignancy even with multiple guesses regardless of the underlying cost functions. Fig. 5 presents the percentages of successful detection and the histograms of sensing time. It can be seen that the GD-inspired CDP yields a $94 \%$ detection ratio for the Sphere function, which is higher than that for the Matyas function (74.8\%) and much higher than random search for both the two cost functions $(\sim 37 \%)$. This observation demonstrates the advantage of the proposed biosensing-by-learning strategy over random search and the potential performance deterioration due to a more complex landscape (i.e., plate-shaped Matyas function versus bowlshaped Sphere function). Secondly, the detection time is distributed between $80 \mathrm{~s}$ and 100s for all the four histograms, whereas the Sphere function in Fig. 5(a) exhibits the most asymmetric distribution and the Matyas function in Fig. 5(b) yields the lowest mean detection time.

\section{CONCLUSION}

We have presented a novel biosensing-by-learning framework. The proposed approach carries out optimized cancer detection inspired by computational algorithms. Specifically, we have proposed the GD-inspired CDP and presented numerical examples to demonstrate the effectiveness of the proposed methodology. We believe that this work motivates

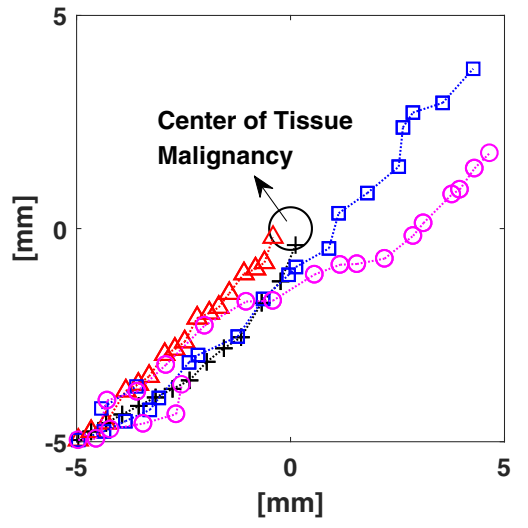

Fig. 4. Trajectories of guess inputs: “+"-Sphere function, " $\triangle$ "-Matyas function, " $\square$ "-random search in a Sphere landscape, "०"-random search in a Matyas landscape.
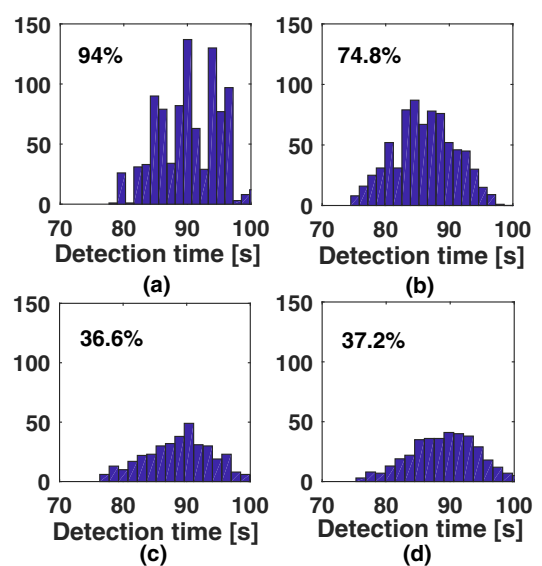

Fig. 5. Histograms of detection time for (a) Sphere function, (b) Matyas function, (c) random search in a Sphere landscape, and (d) random search in a Matyas landscape. Also shown are the respective detection ratios.

a new paradigm directed toward smart biosensing facilitated by externally controllable nanorobots.

\section{REFERENCES}

[1] Y. Chen, et al., "Green touchable nanorobotic sensor networks," IEEE Commun. Mag., vol. 54, no. 11, pp. 136-142, 2016.

[2] Y. Chen, et al., "Touchable computing: Computing-inspired biodetection," IEEE Trans. Nanobiosci., vol. 16, no. 8, pp. 810-821, 2017.

[3] O. Felfoul et al., "Magneto-aerotactic bacteria deliver drug-containing nanoliposomes to tumour hypoxic regions," Nat. Nanotechnol., vol. 11, pp. 941-947, 2016.

[4] G. von Maltzahn et al., "Nanoparticles that communicate in vivo to amplify tumour targeting," Nat. Mater., vol. 10, no. 7, pp. 545-552, 2012.

[5] J. W. Baish et al., "Role of tumor vasculature architecture in nutrient and drug delivery: An invasion percolation-based network model," Microvascular Res., vol. 51, pp. 327-346, 1996.

[6] D.-S. Lee, H. Rieger, and K. Bartha, "Flow correlated percolation during vascular remodeling in growing tumors," Phys. Rev. Lett., vol. 96, nos. 5-10, pp. 058104, 2006.

[7] Y. Chen, et al., "A touch-communication framework for drug delivery based on a transient microbot system," IEEE Trans. Nanobiosci., vol. 14, no. 4, pp. 397-408, 2015.

[8] J. Barzilai and J. M. Borwein, "Two point step size gradient methods," IMA J. Numer. Anal., vol. 8, pp. 141-148, 1988. 